\title{
CAPITULATION IN UNRAMIFIED QUADRATIC EXTENSIONS OF REAL QUADRATIC NUMBER FIELDS
}

\author{
by E. BENJAMIN, F. SANBORN and C. SNYDER
}

(Received 8 March, 1993; revised 21 May, 1993)

1. Introduction. Let $k$ be an algebraic number field and $C_{k}$ its ideal class group (in the wider sense). Suppose $K$ is a finite extension of $k$. Then we say that an ideal class of $k$ capitulates in $K$ if this class is in the kernel of the homomorphism

$$
j: C_{k} \rightarrow C_{K}
$$

induced by extension of ideals from $k$ to $K$. (See Section 2 below). In [4], Iwasawa gives examples of real quadratic number fields, $k=\mathbb{Q}\left(\sqrt{p_{1} p_{2} p_{3}}\right)$, with distinct primes $p_{i} \equiv 1$ $(\bmod 4)$, for which all the ideal classes of the 2-class group, $C_{k, 2}$ (the 2-Sylow subgroup of $C_{k}$ ), capitulate in an unramified quadratic extension of $k$. In these examples, $C_{k, 2}$ is abelian of type $(2,2)$, i.e. isomorphic to $\mathbb{Z} / 2 \mathbb{Z} \times \mathbb{Z} / 2 \mathbb{Z}$, and so all four ideal classes capitulate.

In this note, we consider an arbitrary unramified quadratic extension, $K / k$, of a real quadratic number field, $k$, and determine the number of ideal classes of $C_{k}$ which capitulate in $C_{K}$. As we shall see, the number of ideal classes that can capitulate is 2,4 , or 8. We give simple criteria involving the fundamental units of the three quadratic subfields of $K$ which determine the number of ideal classes that capitulate. We then make use of the results of Cremona and Odoni $[1,2]$ to show that there exist infinitely many extensions $K / k$ such that $|\operatorname{ker} j|=2,4$, and 8 respectively. Examples are then provided.

For more information on the capitulation problem, see Miyake [7].

2. Main Results. Let $k$ be a real quadratic number field of even class number with discriminant

$$
d_{k}=d=p_{1}^{*} \ldots p_{r}^{*}, \quad p_{j} \text { distinct primes. }
$$

Here $p^{*}$ represents the fundamental discriminant divisible only by the prime $p$, i.e. $p^{*}=(-1)^{p-1 / 2} p$, if $p$ is odd, and $2^{*} \in\{-4,8,-8\}$.

Since the class number of $k$ is even, there exists at least one quadratic extension, $K$, of $k$ unramified at all the primes (including the infinite ones, which means $K$ is totally real). By genus theory, see e.g. [5], $K=k\left(\sqrt{d_{1}}\right)$ for some fundamental discriminant $d_{1} \mid d$ such that $d_{1}>1$ and $d_{1} \neq d$. Let $d_{2}=d / d_{1}$. Then $K=\mathbb{Q}\left(\sqrt{d_{1}}, \sqrt{d_{2}}\right)$ and thus $K / \mathbb{Q}$ is a Galois extension, the Galois group of which is abelian of type $(2,2)$. Consequently, $K$ contains three real quadratic subfields: $k_{0}=k=\mathbb{Q}(\sqrt{d}), k_{1}=\mathbb{Q}\left(\sqrt{d_{1}}\right)$ and $k_{2}=\mathbb{Q}\left(\sqrt{d_{2}}\right)$. We have the following diagram.

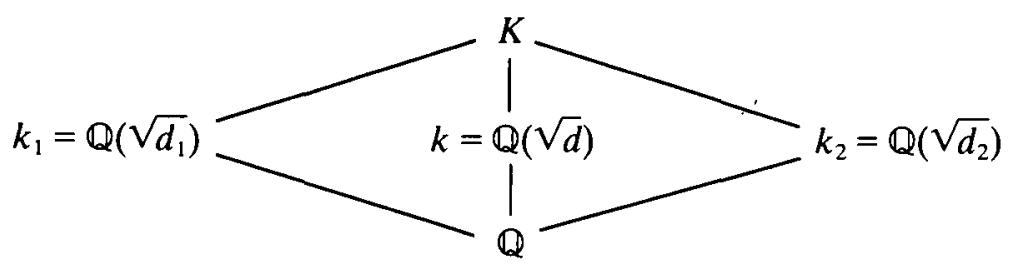

Glasgow Math. J. 36 (1994) 385-392. 
We denote by $\varepsilon_{0}=\varepsilon, \varepsilon_{1}$, and $\varepsilon_{2}$ the fundamental units $(>1)$ of $k_{0}=k, k_{1}$, and $k_{2}$, respectively. Also to simplify notation we let $N \kappa_{i}=N_{k_{i} \mathbb{Q Q}}\left(\kappa_{i}\right)$ for any $\kappa_{i} \in k_{i}(i=0,1,2)$. Thus $N \kappa_{i}=\kappa_{i} \kappa_{i}^{\prime}$ where $\kappa_{i}^{\prime}$ denotes the conjugate of $\kappa_{i}$ over $\mathbb{Q}$.

Let $j: C_{k} \rightarrow C_{K}$ be the homomorphism induced by extension of ideals from the ring of integers, $O_{k}$, of $k$ to $O_{K}$. (Hence if $[A]$ is the ideal class of $C_{k}$ containing the ideal $A$, then $j([A])=\left[A O_{K}\right]$.) We shall be interested in determining $\mid$ ker $j \mid$, the number of ideal classes of $C_{k}$ that capitulate in $C_{K}$. It is well-known, cf. [3], that the exponent of the group ker $j$ divides $[K: k]$ (which for us is 2). Hence $\operatorname{ker} j \subseteq C_{k .2}$, the 2-Sylow subgroup of $C_{k}$. Moreover it is also well-known, cf. [9], that when $K / k$ is cyclic and unramified at the infinite primes,

$$
|\operatorname{ker} j|=[K: k]\left[E_{k}: N_{K / k}\left(E_{K}\right)\right] .
$$

Hence in our case $|\operatorname{ker} j|=2\left[E_{k}: N_{K / k}\left(E_{K}\right)\right]$. Here $E_{k}, E_{K}$ represent the group of units in $O_{k}, O_{K}$, respectively. Notice that $E_{k}^{2} \subseteq N_{K / k}\left(E_{K}\right)$. But then since $E_{k}=\{ \pm 1\} \times\langle\varepsilon\rangle$, we see that

$$
\left[E_{k}: N_{K / k}\left(E_{K}\right)\right] \leq\left[E_{k}: E_{k}^{2}\right]=4 .
$$

Thus $\left[E_{k}: N_{K / k}\left(E_{K}\right)\right]=1,2$, or 4 and so $|\operatorname{ker} j|=2,4$, or 8 . We now determine conditions under which each of these three possibilities can happen. To this end, we obtain more information about $E_{K}$.

By Dirichlet's unit theorem, since $[K: \mathbb{Q}]=4$ and $K$ is totally real, $E_{K}$ possesses a system of three fundamental units, i.e. $E_{K}=\{ \pm 1\} \times\left\{\mu_{1}\right\} \times\left\{\mu_{2}\right\} \times\left\{\mu_{3}\right\}$ for some $\mu_{i} \in$ $E_{K}(i=1,2,3)$.

By Kubota [6, Satz 1], there exist the following eight possibilities for a system of fundamental units of $E_{K}$ :
(i) $\varepsilon_{i}, \varepsilon_{j}, \varepsilon_{k}$
(ii) $\sqrt{\varepsilon_{i}}, \varepsilon_{j}, \varepsilon_{k}$
(iii) $\sqrt{\varepsilon_{i}}, \sqrt{\varepsilon_{j}}, \varepsilon_{k}$
(iv) $\sqrt{\varepsilon_{i} \varepsilon_{j}}, \varepsilon_{j}, \varepsilon_{k}$
(vi) $\sqrt{\varepsilon_{i} \varepsilon_{j}}, \sqrt{\varepsilon_{j} \varepsilon_{k}}, \sqrt{\varepsilon_{k} \varepsilon_{i}}$
(v) $\sqrt{\varepsilon_{i} \varepsilon_{j}}, \sqrt{\varepsilon_{k}}, \varepsilon_{j}$
(vii) $\sqrt{\varepsilon_{i} \varepsilon_{j} \varepsilon_{k}}, \varepsilon_{j}, \varepsilon_{k}$
(viii) $\sqrt{\varepsilon_{i} \varepsilon_{j} \varepsilon_{k}}, \varepsilon_{j}, \varepsilon_{k}$ (with $N \varepsilon_{l}=-1(l=0,1,2)$ )

where $\left\{\varepsilon_{i}, \varepsilon_{j}, \varepsilon_{k}\right\}=\left\{\varepsilon_{0}, \varepsilon_{1}, \varepsilon_{2}\right\}$. Also in cases (ii)-(vii), any $\varepsilon_{t}$ that appears under a radical is assumed to have norm equal to 1 .

Proposition 1. Suppose $N \varepsilon_{i}=-1$ for $i=0,1,2$. Then

$$
\left[E_{k}: N_{K / k}\left(E_{K}\right)\right]= \begin{cases}1 & \text { if } \sqrt{\varepsilon_{0} \varepsilon_{1} \varepsilon_{2}} \in K, \\ 2 & \text { otherwise. }\end{cases}
$$

Remark. Using [6, Zusatz 1], it is easy to determine whether $\sqrt{\varepsilon_{0} \varepsilon_{1} \varepsilon_{2}} \in K$. See the first example of Section 3.

Proof of the Proposition. Since $N_{K / k}\left(\varepsilon_{1}\right)=N_{k_{1} / Q}\left(\varepsilon_{1}\right)=N \varepsilon_{1}=-1$, we have $-1 \in$ $N_{K / k}\left(E_{K}\right)$. If $\sqrt{\varepsilon_{0} \varepsilon_{1} \varepsilon_{2}} \in K$, then $N_{K / k}\left(\sqrt{\varepsilon_{0} \varepsilon_{1} \varepsilon_{2}}\right)= \pm \varepsilon_{0}$ (because $\left(N_{K / k} \sqrt{\varepsilon_{0} \varepsilon_{1} \varepsilon_{2}}\right)^{2}=$ $\left.N_{K / k}\left(\varepsilon_{0} \varepsilon_{1} \varepsilon_{2}\right)=\varepsilon_{0}^{2} N \varepsilon_{1} N \varepsilon_{2}=\varepsilon_{0}^{2}\right)$. Thus $\pm \varepsilon_{0} \in N_{K / k}\left(E_{K}\right)$ and so $\left[E_{k}: N_{K / k}\left(E_{K}\right)\right]=1$.

Now if $\sqrt{\varepsilon_{0} \varepsilon_{1} \varepsilon_{2}} \notin K$, then $\varepsilon_{0}, \varepsilon_{1}, \varepsilon_{2}$ must be a system of fundamental units of $E_{K}$ and thus $N_{K / k}\left(E_{K}\right)=\{ \pm 1\} \times\left\{\varepsilon_{0}^{2}\right\}$ which is of index 2 in $E_{k}$. This establishes the proposition.

Proposition 2. Suppose $N \varepsilon_{i}=1$ for some $i=0,1,2$. Furthermore suppose $N \varepsilon_{1}=-1$ 
or $N \varepsilon_{2}=-1$. (Without loss of generality assume $N \varepsilon_{2}=-1$ ). Then

$$
\left[E_{k}: N_{K / k}\left(E_{K}\right)\right]= \begin{cases}1 & \text { if } \sqrt{\varepsilon_{0}} \in K \text { or } \sqrt{\varepsilon_{0} \varepsilon_{1}} \in K, \\ 2 & \text { otherwise. }\end{cases}
$$

In particular, if $N \varepsilon_{0}=-1$, then $\left[E_{k}: N_{K / k}\left(E_{K}\right)\right]=2$. [6].

REMARK. We shall see that the condition $\sqrt{\varepsilon_{0}}, \sqrt{\varepsilon_{0} \varepsilon_{1}} \in K$ is easy to check by Kubota

Proof of the Proposition. Suppose $\sqrt{\varepsilon_{0}} \in K$ or $\sqrt{\varepsilon_{0} \varepsilon_{1}} \in K$. Then $N_{K / k}\left(\sqrt{\varepsilon_{0} \varepsilon_{1}^{a}}\right)= \pm \varepsilon_{0}$ ( $a=0$ or 1) (arguing as in the proof of Proposition 1). Moreover, $N_{K / k}\left(\varepsilon_{2}\right)=N \varepsilon_{2}=-1$. Hence $E_{k}=N_{K / k}\left(E_{K}\right)$ establishing part of the proposition.

Now suppose $\sqrt{\varepsilon_{0}} \notin K$ and $\sqrt{\varepsilon_{0} \varepsilon_{1}} \notin K$. Then by Kubota [6, Satz 1] (cf. above) a system of fundamental units of $E_{K}$ consists of $\varepsilon_{0}, \varepsilon_{1}, \varepsilon_{2}$ or perhaps $\varepsilon_{0}, \sqrt{\varepsilon_{1}}, \varepsilon_{2}$. (Note that any unit, $\varepsilon_{i}$, under a radical must have positive norm). In either case, $N_{K / k}\left(E_{K}\right)=\left\langle-1, \varepsilon_{0}^{2}\right\rangle$ which is of index 2 in $E_{k}$. This establishes the proposition.

In Proposition 3 below, we consider the case that $N \varepsilon_{1}=N \varepsilon_{2}=1$. As this case requires more effort we first single out a major concept.

Definition. Suppose $\mu$ is a unit of a real quadratic field such that $N \mu=1$. We define, as in [6], $\delta(\mu)$ as the square-free kernel of the rational integer $\mu+\mu^{\prime}+2$, i.e. if $\mu+\mu^{\prime}+2=m^{2} n$ for some integers $m, n$ and $n$ is square-free, then $\delta(\mu)=n$.

For convenience we isolate facts about $\delta$ found in [6].

LEMMA. Let $L$ be a noncyclic normal real quartic extension of $\mathbb{Q}$ containing three real quadratic fields $k_{1}, k_{2}$, and $k_{3}$. Suppose $\eta_{i} \in E_{k_{i}}$ with $N \eta_{i}=1$ for $i=1,2,3$. Then

(1) $\delta\left(\eta_{i}\right) \mid d_{k_{i}}$

(2) $\eta_{1} \eta_{2} \eta_{3} \in E_{L}^{2}$ (the squares in $\left.E_{L}\right)$ iff $\delta\left(\eta_{1}\right) \delta\left(\eta_{2}\right) \delta\left(\eta_{3}\right) \in L^{2}$.

Also if $k$ is any real quadratic field such that $k=\mathbb{Q}(\sqrt{\Delta})$ with $\Delta$ square-free and such that $N(\varepsilon)=1$ for the fundamental unit $\varepsilon$, then

(3) $\delta(\varepsilon) \neq 1, \Delta$.

Proof. See Kubota [6], Hilfssätze 8, 11, and 9, respectively.

Proposition 3. Suppose $N \varepsilon_{1}=N \varepsilon_{2}=1$. Then

$$
\left[E_{k}: N_{K / k}\left(E_{K}\right)\right]= \begin{cases}2 & \text { if } \sqrt{\varepsilon_{0} \varepsilon_{1}^{a} \varepsilon_{2}^{b}} \in K \text { some } a, b \in\{0,1\} \\ 4 & \text { otherwise. }\end{cases}
$$

In particular, if $N \varepsilon_{0}=-1$, then $\left[E_{k}: N_{K / k}\left(E_{K}\right)\right]=4$.

Proof. We first claim that $\sqrt{\varepsilon_{1}}, \sqrt{\varepsilon_{2}}, \sqrt{\varepsilon_{1} \varepsilon_{2}} \notin K$. To this end let $k_{i}=\mathbb{Q}\left(\sqrt{\Delta_{i}}\right)$ $(i=0,1,2)$ where $\Delta=\Delta_{0}, \Delta_{1}, \Delta_{2}$ are square-free rational integers. Also let $\delta_{i}=\delta\left(\varepsilon_{i}\right)$ for $i=1,2$ and $\delta_{0}=\delta\left(\varepsilon_{0}\right)$ if $N \varepsilon_{0}=1$. Notice that $K=\mathbb{Q}\left(\sqrt{\Delta_{1}}, \sqrt{\Delta_{2}}\right)$. We show that $\delta_{1}, \delta_{2}$, $\delta_{1} \delta_{2} \notin K^{2}$. We do this by considering three cases according as $\Delta \equiv 1,-1,2(\bmod 4)$, respectively. Also in each case $p_{i}(i=1, \ldots, s)$ denote distinct primes $\equiv 1(\bmod 4)$ and $q_{j}(j=1, \ldots, t)$ distinct primes $\equiv-1(\bmod 4)$.

Case 1. Suppose $\Delta \equiv 1(\bmod 4)$. Then $d=\Delta$. 
Let $d=p_{1} \ldots p_{s} q_{1} \ldots q_{r}=\Delta$ with $t$ even. ( $s=0$ or $t=0$ is possible.) lemma,

Also let $d_{1}=p_{1} \ldots p_{s_{1}} q_{1} \ldots q_{t_{1}}=\Delta_{1}$ with $t_{1}$ even. Then $d_{2}=d / d_{1}=\Delta_{2}$. By the

$$
\begin{array}{lll}
\delta_{1} \mid d_{1}=\Delta_{1} & \text { and } & \delta_{1} \neq 1, \Delta_{1} \\
\delta_{2} \mid d_{2}=\Delta_{2} & \text { and } & \delta_{2} \neq 1, \Delta_{2}
\end{array}
$$

Hence $\delta_{1}, \delta_{2}, \delta_{1} \delta_{2} \notin K^{2}$.

Case 2. Suppose $\Delta \equiv-1(\bmod 4)$. Then $d=4 \Delta$.

Let $\Delta=p_{1} \ldots p_{s} q_{1} \ldots q_{1}$ with $t$ odd. Without loss of generality, let $\Delta_{1}=$ $p_{1} \ldots p_{s_{1}} q_{1} \ldots q_{t_{1}}$ with $t_{1}$ odd and so $d_{1}=4 \Delta_{1}$. Then $d_{2}=d / d_{1}=\Delta / \Delta_{1}=\Delta_{2}$.

By the lemma, $\delta_{2} \mid d_{2}=\Delta_{2}$ and $\delta_{2} \neq 1, \Delta_{2}$; hence $\delta_{2} \notin K^{2}$. On the other hand, $\delta_{1} \mid d_{1}=4 \Delta_{1}$ and $\delta_{1} \neq 1, \Delta_{1}$. Thus since $\delta_{1}$ is square-free $\delta_{1}=2,2 \Delta_{1}, a_{1}$, or $2 a_{1}$ for some $a_{1} \mid \Delta_{1}, a_{1} \neq 1, \Delta$. If $\delta_{1}=a_{1}$ or $2 a_{1}$, then $\delta_{1}, \delta_{1} \delta_{2} \notin K^{2}$. If $\delta_{1}=2$ or $2 \Delta_{1}$, then the only way $\delta_{1} \in K^{2}$ can occur is if $\sqrt{2} \in K$. But then $k_{i}=\mathbb{Q}(\sqrt{2})$ for some $i=1$, 2, which is contrary to the assumption that $N \varepsilon_{1}=N \varepsilon_{2}=1$. Thus $\delta_{1}, \delta_{2}, \delta_{1} \delta_{2} \notin K^{2}$.

Case 3: Suppose $\Delta \equiv 2(\bmod 4)$. Then $d=4 \Delta$.

Let $\Delta=2 p_{1} \ldots p_{s} q_{1} \ldots q_{r}$. Without loss of generality, let $\Delta_{1}=2 p_{1} \ldots p_{s_{1}} q_{1} \ldots q_{r_{1}}$ with $t_{1} \equiv t(\bmod 2)$ and so $d_{1}=4 \Delta_{1}$. Then $d_{2}=d / d_{1}=\Delta / \Delta_{1}=\Delta_{2}$.

The argument of Case 2 now applies and we see once again that $\delta_{1}, \delta_{2}, \delta_{1} \delta_{2} \notin K^{2}$.

Thus by the lemma we see $\sqrt{\varepsilon_{1}}, \sqrt{\varepsilon_{2}}, \sqrt{\varepsilon_{1} \varepsilon_{2}} \notin K$.

Now by Kubota [6, Satz 1] and by our claim we have the following possibilities for a system of fundamental units in $E_{K}$ :

(i) $\varepsilon_{0}, \varepsilon_{1}, \varepsilon_{2}$

(ii) $\sqrt{\varepsilon_{0}}, \varepsilon_{1}, \varepsilon_{2}$

(iv) $\sqrt{\varepsilon_{0} \varepsilon_{1}}, \varepsilon_{i}, \varepsilon_{2}(i=0$ or 1$)$ or $\sqrt{\varepsilon_{0} \varepsilon_{2}}, \varepsilon_{1}, \varepsilon_{i}(i=0$ or 2$)$

(vii) $\sqrt{\varepsilon_{0} \varepsilon_{1} \varepsilon_{2}}, \varepsilon_{i}, \varepsilon_{j}(i$ or $j \in\{1,2\})$.

From this list it follows that if $\sqrt{\varepsilon_{0} \varepsilon_{1}^{a} \varepsilon_{2}^{b}} \in K$, then $\left[E_{k}: N_{K / k}\left(E_{K}\right)\right]=2$, whereas if not, then we are in case (i) in which case $\left[E_{K}: N_{K / k}\left(E_{K}\right)\right]=4$.

This establishes the proposition.

We summarize our results in the following theorem.

THEOREM. Let $K$ be an unramified quadratic extension of a real quadratic number field k. Then

(1) $|\operatorname{ker} j|=2 \Leftrightarrow$ (a) $N \varepsilon_{i}=-1$ for $i=0,1,2$ and $\sqrt{\varepsilon_{0} \varepsilon_{1} \varepsilon_{2}} \in K$ or (b) $\left(N \varepsilon_{1}=-1\right.$ or $\left.N \varepsilon_{2}=-1\right)$ and $N \varepsilon_{0}=1$ and $\left(\sqrt{\varepsilon_{0}}\right.$ or $\sqrt{\varepsilon_{0} \varepsilon_{1}}$ or $\left.\sqrt{\varepsilon_{0} \varepsilon_{2}} \in K\right)$.

(2) $|\operatorname{ker} j|=8 \Leftrightarrow$ (a) $N \varepsilon_{1}=N \varepsilon_{2}=1$ and $N \varepsilon_{0}=-1$ or (b) $N \varepsilon_{i}=1$ for $i=0,1,2$ and $\sqrt{\varepsilon_{0} \varepsilon_{1}^{a} \varepsilon_{2}^{b}} \notin K$ for all $a, b \in\{0,1\}$.

(3) $|\operatorname{ker} j|=4 \Leftrightarrow$ anything else occurs.

Proposition 4. There exist infinitely many real quadratic fields $k$ for which there exists an unramified quadratic extension $K$ in which $|\mathrm{ker} j|=2,4$, and 8 , respectively.

Proof. First consider $|\operatorname{ker} j|=2$. Let $k=\mathbb{Q}\left(\sqrt{p_{1} p_{2}}\right)$ where $p_{i} \equiv 1(\bmod 4)$. Then by genus theory, $C_{k, 2}$ is cyclic and nontrivial. Since the ker $j$ is a nontrivial elementary subgroup of $C_{k, 2}$, it follows that $|\operatorname{ker} j|=2$. Obviously there are infinitely many such fields $k$.

Next consider $|\operatorname{ker} j|=4$. Let $k=\mathbb{Q}\left(\sqrt{p_{1} p_{2} p_{3}}\right)$, where $p_{i} \equiv 1(\bmod 4)$. Then by genus theory, $C_{k, 2}$ has 2-rank equalling 2 and so $|\operatorname{ker} j|=2$ or 4 since $\operatorname{ker} j$ is nontrivial and 
elementary. Hence by our theorem, if we can choose $p_{1}, p_{2}, p_{3}$ such that $N \varepsilon_{0}=-1$ and $N \varepsilon_{1}=1$, then $\mid$ ker $j \mid=4$. To this end choose $k=\mathbb{Q}(\sqrt{(5)(41) p}$ where $p \equiv 3(\bmod 205)$ and $p \equiv 1(\bmod 4)$. Then we claim if $K=k(\sqrt{p})$, then $|\operatorname{ker} j|=4$. For let $k_{1}=\mathbb{Q}(\sqrt{205})$. Then $N \varepsilon_{1}=1$. Moreover the graph $\gamma(5,41)$ is

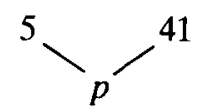

since for $p \equiv 3(\bmod 205),\left(\frac{p}{5}\right)=\left(\frac{p}{41}\right)=-\left(\frac{41}{5}\right)=-1$. (See [1] for the relevant definitions about graphs.) Thus, since $\gamma(5,41, p)$ is odd, Proposition 1.1 of [1] implies $N \varepsilon_{0}=-1$. There are obviously infinitely many such $k$.

Finally consider $|\operatorname{ker} j|=8$. Let $k=\mathbb{Q}\left(\sqrt{p_{1} p_{2} p_{3} p_{4}}\right), p_{i} \equiv 1(\bmod 4)$. If we are able to choose $K=\mathbb{Q}\left(\sqrt{p_{1} p_{2}}, \sqrt{p_{3} p_{4}}\right)$ with $k_{1}=\mathbb{Q}\left(\sqrt{p_{1} p_{2}}\right)$ and $k_{2}=\mathbb{Q}\left(\sqrt{p_{3} p_{4}}\right)$ such that $N \varepsilon_{0}=$ -1 and $N \varepsilon_{1}=N \varepsilon_{2}=1$, then our theorem implies that $\mid$ ker $j \mid=8$. We begin by letting $p_{1}=13, p_{2}=17, p_{3}=5, p_{4}=p$ such that $p \equiv 2(\bmod 13 \times 17)$ and $p \equiv 1(\bmod 5)$. Then the graph $\gamma(13,17,5, p)$ is

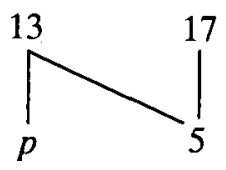

since $\left(\frac{13}{17}\right)=\left(\frac{p}{5}\right)=\left(\frac{p}{17}\right)=-\left(\frac{p}{13}\right)=-\left(\frac{13}{5}\right)=1$. Notice that this graph is odd and thus $N \varepsilon_{0}=-1$. Moreover, $N \varepsilon_{1}=1$. We now need to put additional restrictions on $p$ to insure that $N \varepsilon_{2}=1$. To this end, write $p=\pi \bar{\pi}$ in $\mathbb{Z}[i]$ with $\pi, \bar{\pi}$ prime and $\pi$ primary, i.e. $\pi \equiv 1\left(\bmod (1+i)^{3}\right)$. Choose such a prime $\pi$ in $\mathbb{Z}[i]$ such that

$$
\begin{gathered}
\pi \equiv 1\left(\bmod (1+i)^{3}\right), \pi \equiv i(\bmod 1+2 i), \pi \equiv i(\bmod 1-2 i), \\
\pi \equiv 1+i(\bmod 13), \pi \equiv 1+i(\bmod 17) .
\end{gathered}
$$

The last two congruences imply $p \equiv 2(\bmod 13 \times 17)$ whereas the first three show that the biquadratic residues

$$
\left(\frac{\pi}{1+2 i}\right)_{4}=\left(\frac{\pi}{1-2 i}\right)_{4}=i
$$

By (2.2) of [8], this implies that $N \varepsilon_{2}=1$. Moreover since the righthand sides of the above congruences determine a ray class modulo the ideal $(1+i)(2210)$ in $\mathbb{Z}[i]$, we conclude by class field theory that there are infinitely many primes $\pi$ of residue class degree one over $\mathbb{Q}$ satisfying the congruences. Hence there are infinitely many $p$ such that $p \equiv 2$ $(\bmod 13 \times 17), p \equiv 1(\bmod 5)$ and such that $N \varepsilon_{2}=1$.

This proves the proposition.

3. Examples. In this section we present examples of $K / k$ in which 2,8 , and 4 ideal classes capitulate, respectively. We follow the format of our theorem.

1. $|\operatorname{ker} j|=2$. 
(a) Let $k=\mathbb{Q}(\sqrt{d})$ where $d=65=(5)(13)$. Let $K=k(\sqrt{5})=\mathbb{Q}(\sqrt{5}, \sqrt{13})$. Let $k_{i}=\mathbb{Q}\left(\sqrt{d_{i}}\right)(i=1,2)$ with $d_{1}=5$ and $d_{2}=13$. Then

$$
\begin{aligned}
\varepsilon=\varepsilon_{0}=8+\sqrt{65}, & N \varepsilon_{0}=-1, \\
\varepsilon_{1}=\frac{1+\sqrt{5}}{2}, & N \varepsilon_{1}=-1, \\
\varepsilon_{2}=\frac{3+\sqrt{13}}{2}, & N \varepsilon_{2}=-1 .
\end{aligned}
$$

Set

$$
\begin{aligned}
& c_{0}=\operatorname{Tr}_{K / Q}\left(\varepsilon_{0} \varepsilon_{1} \varepsilon_{2}+\varepsilon_{0}+\varepsilon_{1}-\varepsilon_{2}\right)=117, \\
& c_{1}=\operatorname{Tr}_{K / Q}\left(\varepsilon_{0} \varepsilon_{1} \varepsilon_{2}+\varepsilon_{0}-\varepsilon_{1}+\varepsilon_{2}\right)=125, \\
& c_{2}=\operatorname{Tr}_{K / \mathbb{Q}}\left(\varepsilon_{0} \varepsilon_{1} \varepsilon_{2}-\varepsilon_{0}+\varepsilon_{1}+\varepsilon_{2}\right)=65, \\
& c_{3}=\operatorname{Tr}_{K / Q}\left(\varepsilon_{0} \varepsilon_{1} \varepsilon_{2}-\varepsilon_{0}-\varepsilon_{1}-\varepsilon_{2}\right)=49 .
\end{aligned}
$$

Since $\sqrt{c_{j}} \in K$ for $j=0, \ldots, 3$, we have $\sqrt{\varepsilon_{0} \varepsilon_{1} \varepsilon_{2}} \in K$ by [6, Zusatz 1].

(b) Let $k=\mathbb{Q}(\sqrt{d})$ where $d=105=(5)(3)(7)$. Let $K=k(\sqrt{5})=\mathbb{Q}(\sqrt{5}, \sqrt{21})$. Let $k_{i}=\mathbb{Q}\left(\sqrt{d_{i}}\right)(i=1,2)$ with $d_{1}=5$ and $d_{2}=21$. Then

$$
\begin{aligned}
\varepsilon=\varepsilon_{0}=41+4 \sqrt{105}, & N \varepsilon_{0}=1, \\
\varepsilon_{1}=\frac{1+\sqrt{5}}{2}, & N \varepsilon_{1}=-1 \\
\varepsilon_{2}=\frac{5+\sqrt{21}}{2}, & N \varepsilon_{2}=1 .
\end{aligned}
$$

Moreover $\varepsilon+\varepsilon^{\prime}+2=84=\left(2^{2}\right)(21)$. Thus $\delta(\varepsilon)=21 \in K^{2}$.

Remark. In these two examples, $C_{k, 2}$ is cyclic and thus since ker $j$ is elementary, we see independently that $|\operatorname{ker} j|=2$.

2. $|\operatorname{ker} j|=8$.

(a) Let $k=\mathbb{Q}(\sqrt{d})$ with $d=77285=(5)(13)(29)(41)$. Let $K=k(\sqrt{205})=\mathbb{Q}(\sqrt{(5)(41)}$, $\sqrt{(13)(29))}$. Let $k_{i}=\mathbb{Q}\left(\sqrt{d_{i}}\right)(i=1,2)$ with $d_{1}=205=(5)(41)$ and $d_{2}=377=(13)(29)$. Then

$$
\begin{aligned}
\varepsilon=\varepsilon_{0}=278+\sqrt{77285}, & N \varepsilon_{0}=-1, \\
\varepsilon_{1}=\frac{43+3 \sqrt{205}}{2}, & N \varepsilon_{1}=1, \\
\varepsilon_{2}=233+12 \sqrt{377}, & N \varepsilon_{2}=1 .
\end{aligned}
$$

(b) Let $k=\mathbb{Q}(\sqrt{d})$, with $\quad d=23205=(3)(7)(5)(13)(17)$. Let $K=k(\sqrt{105})=$ $\mathbb{Q}\left(\sqrt{(3)(7)(5)}, \sqrt{(13)(17))}\right.$. Let $k_{i}=\mathbb{Q}\left(\sqrt{\left(d_{i}\right)} \quad(i=1,2)\right.$, with $d_{1}=105=(3)(7)(5)$ and 
$d_{2}=221=(13)(17)$. Then

$$
\begin{aligned}
\varepsilon=\varepsilon_{0}=\frac{457+3 \sqrt{23205}}{2}, & N \varepsilon_{0}=1, \\
\varepsilon_{1}=41+4 \sqrt{105}, & N \varepsilon_{1}=1, \\
\varepsilon_{2}=\frac{15+\sqrt{221}}{2}, & N \varepsilon_{2}=1 .
\end{aligned}
$$

Moreover

$$
\begin{aligned}
\varepsilon+\varepsilon^{\prime}+2 & =459=\left(3^{2}\right)(3)(17), \text { implying } \delta_{0}=\delta(\varepsilon)=(3)(17), \\
\varepsilon_{1}+\varepsilon_{1}^{\prime}+2 & =84=\left(2^{2}\right)(3)(7), \text { implying } \delta_{1}=\delta\left(\varepsilon_{1}\right)=(3)(7), \\
\varepsilon_{2}+\varepsilon_{2}^{\prime}+2 & =17, \text { implying } \delta_{2}=\delta\left(\varepsilon_{2}\right)=17 .
\end{aligned}
$$

Notice that $\delta_{0}, \delta_{0} \delta_{1}, \delta_{0} \delta_{2}, \delta_{0} \delta_{1} \delta_{2} \notin K^{2}$.

3. $|\operatorname{ker} j|=4$.

(i) Let $k=\mathbb{Q}(\sqrt{\mathrm{d}})$, with $d=77285=(5)(13)(29)(41)$ (as in 2.a). Let $K=k(\sqrt{1885})=$ $\mathbb{Q}(\sqrt{(5)(13)(29)}, \sqrt{41})$. Let $k_{i}=\mathbb{Q}\left(\sqrt{d_{i}}\right)(i=1,2)$, with $d_{1}=1885=(5)(13)(29)$ and $d_{2}=$ 41. Then

$$
\begin{aligned}
\varepsilon & =\varepsilon_{0}=278+\sqrt{77285}, & & N \varepsilon_{0}=-1, \\
\varepsilon_{1} & =521+12 \sqrt{1885}, & & N \varepsilon_{1}=1, \\
\varepsilon_{2} & =32+5 \sqrt{41}, & & N \varepsilon_{2}=-1 .
\end{aligned}
$$

By Proposition 2, $\mid$ ker $j \mid=4$.

(ii) Let $k=\mathbb{Q}(\sqrt{d})$, with $d=4641=(3)(7)(13)(17)$. Let $K=k(\sqrt{21})=\mathbb{Q}(\sqrt{(3)(7)}$, $\sqrt{(13)(17)})$. Let $k_{i}=\mathbb{Q}\left(\sqrt{d_{i}}\right)(i=1,2)$, with $d_{1}=21$ and $d_{2}=221=(13)(17)$. Then

$$
\begin{array}{ll}
\varepsilon=\varepsilon_{0}=545+8 \sqrt{4641}, & N \varepsilon_{0}=1, \\
\varepsilon_{1}=\frac{5+\sqrt{21}}{2}, & N \varepsilon_{1}=1, \\
\varepsilon_{2}=\frac{15+\sqrt{221}}{2}, & N \varepsilon_{2}=1 .
\end{array}
$$

Moreover $\varepsilon+\varepsilon^{\prime}+2=1092=\left(2^{2}\right)(3)(7)(13)$, implying $\delta_{0}=\delta(\varepsilon)=(3)(7)(13)$,

$$
\begin{aligned}
& \varepsilon_{1}+\varepsilon_{1}^{\prime}+2=7, \text { implying } \delta_{1}=\delta\left(\varepsilon_{1}\right)=7, \\
& \varepsilon_{2}+\varepsilon_{2}^{\prime}+2=17, \text { implying } \delta_{2}=\delta\left(\varepsilon_{2}\right)=17 .
\end{aligned}
$$

Since $\delta_{0} \delta_{2} \in K^{2}$, Proposition 3 shows $|\mathrm{ker} j|=4$.

Acknowledgements. The authors would like to thank Professor R. W. K. Odoni for suggesting the results in Proposition 4. 


\section{REFERENCES}

1. J. E. Cremona, and R. W. K. Odoni. Some density results for negative Pell equations; an application of graph theory, J. London Math. Soc. (2) 39 (1989), 16-28.

2. J. E. Cremona and R. W. K. Odoni. A generalization of a result of Iwasawa on the capitulation problem, Math. Proc. Cambridge Phil. Soc. 107 (1990), 1-3.

3. Ph. Furtwängler, Über die Klassenzahl abelscher Zahlkörper, J. reine angew. Math. 134 (1908), 91-94.

4. K. Iwasawa, A note on the capitulation problem for number fields, Proc. Japan Acad. Ser. A. Math. Sci. 65 (1989), 59-61.

5. G. Janusz, Algebraic Number Fields, (Academic Press, New York, London, 1973). $65-85$.

6. T. Kubota, Über den bizyklischen biquadratischen Zahlkörpern, Nagoya Math. J. 10 (1956),

7. K. Miyake, Algebraic investigations of Hilbert's Theorem 94, the principal ideal theorem, and the capitulation problem, Expo. Math. 7 (1989), 289-346.

8. R. W. K. Odoni, A note on a recent paper of Iwasawa on the capitulation problem, Proc. Japan Acad. Ser. A. Math. Sci. 65 (1989), 180-182.

9. M. Rosen, Two theorems on Galois cohomology, Proc. Amer. Math. Soc. 17 (1966), $1183-1185$.
E. Benjamin
F. Sanborn and C. Snyder
Department of Mathematics
Department of Mathematics
Unity College
UNIVERSITY OF MAINE
UNITY, MAINE 04988
Orono, MAINE 04469-5752
USA
USA 